\title{
Passively Q-Switched Erbium-Doped Fiber Laser via Evanescent Field Interaction with Few-Layer Molybdenum Ditelluride
}

\author{
Man Wu $(\mathbb{D}$ and Ying Li \\ SZU-NUS Collaborative Innovation Center for Optoelectronic Science \& Technology, International Collaborative Laboratory of \\ 2D Materials for Optoelectronics Science and Technology of Ministry of Education, College of Optoelectronic Engineering, \\ Shenzhen University, Shenzhen 518060, China \\ Correspondence should be addressed to Man Wu; manmwu@szu.edu.cn
}

Received 22 June 2018; Accepted 9 September 2018; Published 1 October 2018

Guest Editor: Xinxing Zhou

Copyright (C) 2018 Man Wu and Ying Li. This is an open access article distributed under the Creative Commons Attribution License, which permits unrestricted use, distribution, and reproduction in any medium, provided the original work is properly cited.

\begin{abstract}
We demonstrate an all-fiberized passively Q-switched erbium-doped fiber laser (EDFL) via evanescent field interaction between molybdenum ditelluride saturable absorber (SA) and guided mode of the D-shaped fiber. By integrating the few-layer molybdenum ditelluride prepared by CVD method onto the side-polished fiber, the SA can be realized by the strong interaction between the evanescent field of the waveguide and the nonlinear optical material. The proposed passively Q-switched EDFL could deliver output pulses at $1566 \mathrm{~nm}$ wavelength with pulse width of $5.03 \mu \mathrm{s}$, a repetition rate of $13.9 \mathrm{kHz}$, a pulse energy of $150.6 \mathrm{~nJ}$, and an output power of $2.1 \mathrm{~mW}$ when pumped by a $980 \mathrm{~nm}$ laser diode of $180 \mathrm{~mW}$.
\end{abstract}

\section{Introduction}

Passively Q-switched fiber lasers have been extensively used in optical communication, optical sensing, industrial processing, etc., for the high beam quality, high stability, high energy, and the compact setups [1-5]. The saturable absorber (SA) is indispensable for the passively Q-switched fiber laser for the quality factor $\mathrm{Q}$ of the optical cavity can be modulated by the intensity-dependent nonlinearity of SAs $[1,2]$. Inspired by the requirements of the compact, cost-effective, stable Q-switched fiber laser, the researchers from optics, optical material, and some other research community have paid much attention on the fields. As we know, the semiconductor saturable absorber mirrors (SESAMs) have been commercialized for the precise control of the absorption wavelength, saturation threshold, modulation depth, and relaxation time. However, SESAMs suffer from the limited bandwidth and complicated preparation procedures [5]. With the emergence of the low-dimensional materials [6-15], especially the twodimensional (2D) nanomaterials $[3,4]$, their unique characteristics, such as the ultrafast recovery time, high damage threshold, and broadband nonlinear optical response, have provided an ideal platform to improve the laser performance. With the remarkable progress of graphene, other $2 \mathrm{D}$ materials, such as transition metal dichalcogenides (TMDs) [8-11], topological insulators $[12,13]$, and black phosphorus $[16,17]$, have also attracted growing research attention resulting from their excellent physical/chemical properties. However, graphene possesses a relatively low modulation depth, and topological insulators are limited to complex preparation processes, and black phosphorus is prone to oxidation and humidity. In contrast to them, TMDs have outstanding layer-dependent semiconducting and nonlinear optical properties.

TMDs, characterized by the chemical formula $\mathrm{MX}_{2}$, where $\mathrm{M}$ is a transition metal (commonly Mo, W) and $\mathrm{X}$ is a group VI element ( $\mathrm{S}, \mathrm{Se}$, and $\mathrm{Te}$ ), are a family of highly anisotropic layered semiconductor materials. For the TMD monolayer, it is composed of two hexagonal planes of $\mathrm{X}$ atoms and an intermediate hexagonal plane of $\mathrm{M}$ atoms coordinated by covalent bonds with the $\mathrm{X}$ atoms in a trigonal prismatic arrangement $[8,18]$. With the technical evolution, the nonlinear optical performance of TMDs, such as $\mathrm{MoS}_{2}, \mathrm{WS}_{2}, \mathrm{MoSe}_{2}$, and $\mathrm{WSe}_{2}$, has been investigated. Compared to other TMDs, telluride material has not attracted adequate attentions. In addition, it is found that $\mathrm{MoTe}_{2}$, in contrast to $\mathrm{MoS}_{2}$ and $\mathrm{MoSe}_{2}$, has smaller bandgap, which implied it can exhibit broadband absorption. Most recently, 


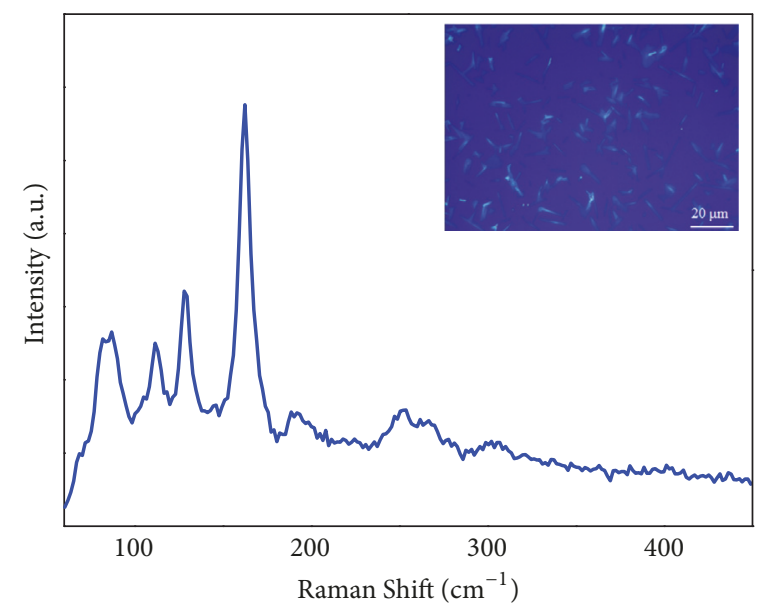

FIGURE 1: Raman spectra of the few-layer $\mathrm{MoTe}_{2}$, and the inset shows its SEM image.

Mao et al. reported the nonlinear optical properties of few-layer $\mathrm{MoTe}_{2}$ fabricated by a liquid exfoliation method and achieved soliton mode-locking operations in an EDFL [18]. Wang et. al. realized the high energy soliton pulse output with magnetron-sputtering-deposition-grown $\mathrm{MoTe}_{2}$ saturable absorber [19]. Liu et. al. have implemented a $\mathrm{MoTe}_{2}-$ based passively Q-switched EDFL via magnetron sputtering technique [20]. Yan et. al. have also investigated the nonlinear optical performance of $\mathrm{MoTe}_{2}$ around $3 \mu \mathrm{m}$ in a solid-state laser system [21]. However, the material quality and laser energy based on $\mathrm{MoTe}_{2}$ still need improvement.

In this contribution, we have prepared the few-layer $\mathrm{MoTe}_{2}$ via the chemical vapor deposition method and fabricated an all-fiberized SA by transferring the $\mathrm{MoTe}_{2}$ onto the D-shaped fiber. With the help of the strong interaction between evanescent field and the nonlinear optical material, the all-fiberized Q-switched fiber laser can be realized, and the high energy pulsed fiber laser can be delivered.

\section{Materials Preparation and Characterizations}

The $\mathrm{MoTe}_{2}$ has been prepared via the chemical vapor deposition method. The quartz boat containing $5 \mathrm{mg}$ Te powders is placed upstream of the tube with a distance of $14 \mathrm{~cm}$ from the heating center, while the second boat containing $0.5 \mathrm{mg}$ $\mathrm{MoO}_{3}$ powders is placed on the heating center of the tube. The substrate is placed above the second boat and faced up. The mixed gas of $\mathrm{H}_{2} / \mathrm{Ar}$ (95\% Ar) is flowing with 100 sccm for $30 \mathrm{~min}$ as the cleaning gas before heating and with $30-100 \mathrm{sccm}$ as the carrier gas during the heating process. The pressure is in atmospheric environment. The furnace is heated to $720^{\circ} \mathrm{C}$ in $20 \mathrm{~min}$ and then maintained at given temperature for $30 \mathrm{~min}$ before it is naturally cooled. Then the few-layer $\mathrm{MoTe}_{2}$ can be prepared.

The Raman spectra of prepared $\mathrm{MoTe}_{2}$ show the fewlayer nature of the material, which agree well with the earlier finding [22]. As shown in the inset of Figure 1, the scanning electron microscope (SEM) images suggest the 2D nature of the $\mathrm{MoTe}_{2}$. With the grown $\mathrm{MoTe}_{2}$, we have characterized its nonlinear absorption performance via the Z-scan technique with a $1560 \mathrm{~nm}$ mode-locked fiber laser whose repetition rate is $\sim 20 \mathrm{MHz}$ and pulse width is $\sim 1.5$ ps. Figure 2(a) shows the typical open-aperture curve of the $\mathrm{MoTe}_{2}$ sample, which manifests that the material can exhibit nonlinear saturable absorption characteristics. In addition, we have given the relationship between incident intensity and the nonlinear transmittance, as shown in Figure 2(b), by fitting the experimental results with the following equation [11]:

$$
\alpha\left(I_{\mathrm{n}}\right)=\frac{\alpha_{\mathrm{s}}}{1+I_{n} / I_{\mathrm{s}}}+\alpha_{\mathrm{ns}}
$$

where $\alpha\left(I_{\mathrm{n}}\right)$ is the absorption coefficient, $\alpha_{\mathrm{s}}$ is the saturable loss, $\alpha_{\text {ns }}$ is nonsaturable absorbance, and $I$ and $I_{\mathrm{s}}$ are input and the saturation intensities, respectively. The fitted modulation depth and saturable intensity are $3 \%$ and $6 \mathrm{MW} / \mathrm{cm}^{2}$, respectively.

\section{Experimental Results and Discussions}

To validate the nonlinear optical response of the $\mathrm{MoTe}_{2}$, we have fabricated a fiberized SA by integrating the $\mathrm{MoTe}_{2}$ onto the D-shaped fiber. Moreover, we followed the methods of $\mathrm{Du}$ et al. [11] to design and build an EDFL with a ring cavity configuration modulated by the $\mathrm{MoTe}_{2} \mathrm{SA}$, as shown in Figure 3. In the experimental setup, the overall cavity length is about $8.6 \mathrm{~m}$, which consists of a piece of high concentration erbium doped fiber (LIEKKI Er80-8/125) with length about $0.8 \mathrm{~m}$, which is pumped by a commercial $980 \mathrm{~nm}$ laser diode (LD), a polarization independent isolator (PIISO) to guarantee the unidirectional laser operation, an intracavity polarization controller (PC) to tune the cavity birefringence, and some other single mode fiber (SMF-28) optical components. We adopted a 980/1550 WDM coupler to introduce the $980 \mathrm{~nm}$ pump laser into the fiber ring cavity. Different from the work with transmission-type SA [11], we introduced a $\mathrm{MoTe}_{2} \mathrm{SA}$ via evanescent field interaction between guided wave and the $\mathrm{MoTe}_{2}$, which can make the whole laser setup compact in an all-fiberized way. In addition, the evanescent field interaction can improve the interaction 


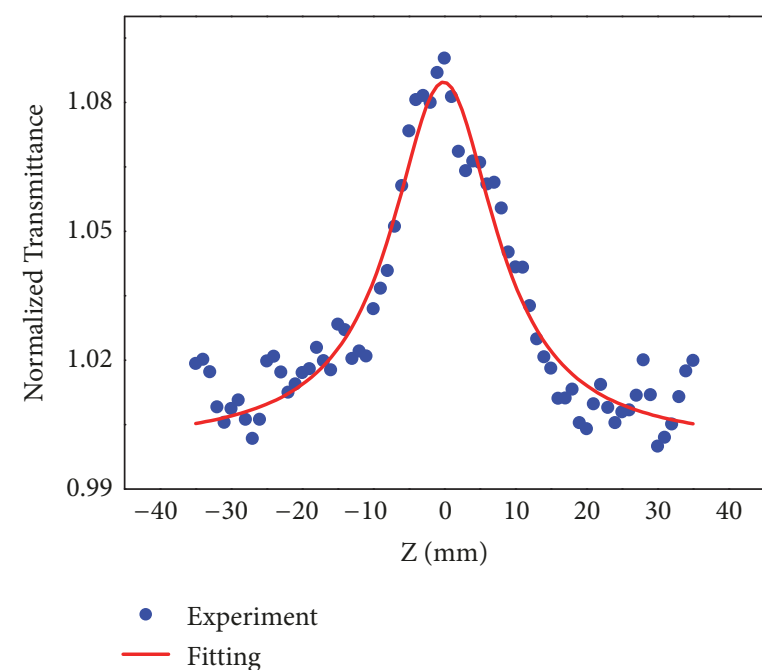

(a)

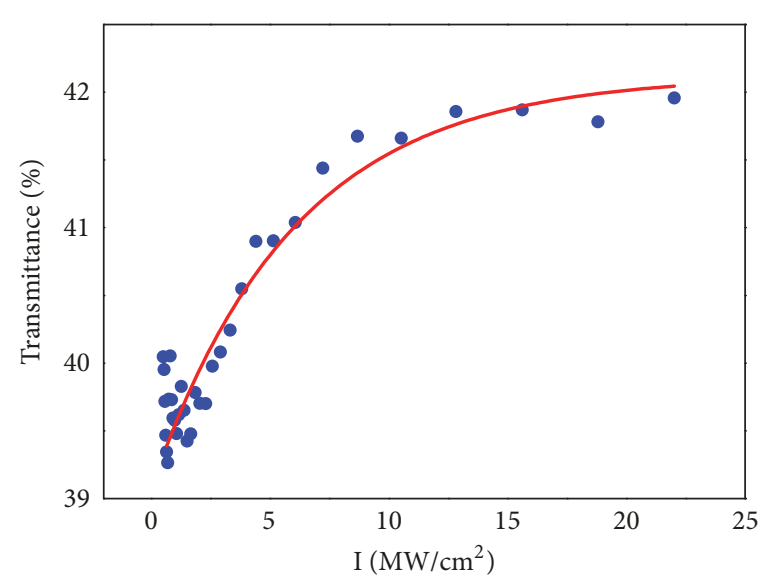

- Experiment Fitting

FIgURE 2: The experimental data of the nonlinear transmittance of the $\mathrm{MoTe}_{2}$.

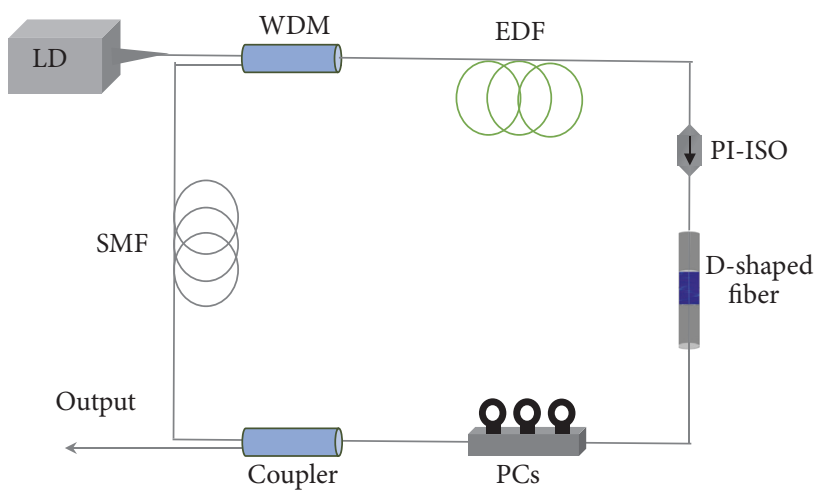

FIgURE 3: The experimental setup of the Q-switched fiber laser based on $\mathrm{MoTe}_{2}$.

length and alleviate the material damage. To monitor the laser performance, an optical spectrum analyser (Ando AQ-6317B) and a real-time oscilloscope with a bandwidth of $4 \mathrm{GHz}$ (Agilent DSO9404A) together with a photodetector (MC PD-12D) have been employed to simultaneously monitor the optical spectrum and the temporal evolution of the output pulse train. In addition, the output power of the Qswitched fiber laser was measured with a power meter (Ando, AQ2140).

In this experiment, the self-started Q-switching operation can be obtained by increasing the pump power up to around $60 \mathrm{~mW}$. Figure 4 summarizes the typical operation characteristics of the Q-switching state at a pump power of $180 \mathrm{~mW}$. Figure 4(a) shows the optical spectrum centered at $1566 \mathrm{~nm}$ delivered from the Q-switched fiber laser. Figure 4(b) shows the typical Q-switched pulse train with a repetition rate of $13.9 \mathrm{kHz}$. It can be seen that no peak intensity modulation had been observed on the pulse train, illustrating the high stability of Q-switching operation. The inset of Figure 4(b) shows the profile of the Q-switched pulse with a symmetric Gaussianlike shape with a full width at half maximum (FWHM) of 5.03 $\mu$ s.
We have also investigated the power-dependent pulse duration, repetition rate, output power, and the pulse energy of the Q-switched fiber laser. Figure 5(a) presents the evolution curves of the repetition rate and pulse duration with the pump power. It shows typical features of the Q-switching operation. By increasing the pump power, the repetition rate will linearly increase from $4.9 \mathrm{kHz}$ to $13.9 \mathrm{kHz}$, while the pulse duration varied in the range of $32.0 \mu \mathrm{s}$ to 5.03 $\mu \mathrm{s}$. Figure 5(b) shows the relationship between the average output power and the pulse energy with the pump power. The average output power increased almost linearly with the pump power. Moreover, at the maximum pump power of $180 \mathrm{~mW}$, the maximum obtained pulse energy is up to 150.6 $\mathrm{nJ}$, which is higher than or comparable to that of the Q-switch pulses obtained in the Er-doped fiber lasers with other SAs, such as CNTs, graphene, and TI, and higher than that of the reported results based on $\mathrm{MoTe}_{2}$ SA [17-19]. The improved pulse energy can result from the effective interaction between the evanescent field of the fiber and the few-layer $\mathrm{MoTe}_{2}$, which can provide a good platform to alleviate the damage threshold and tune the linear or nonlinear interaction with an all-fiberized configuration. Although the microfiber can 


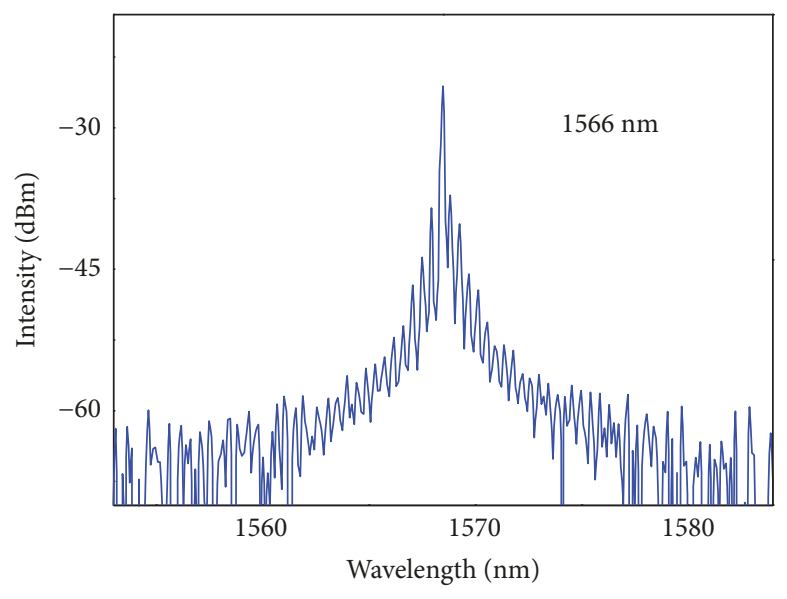

(a)

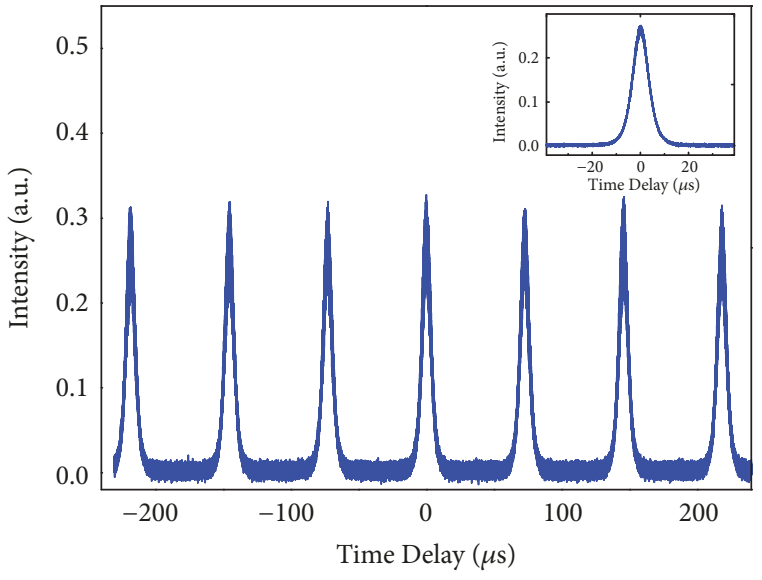

(b)

FIgURE 4: (a) The spectra of the Q-switched fiber laser based on $\mathrm{MoTe}_{2}$. (b) The pulse train and the profile of the Q-switched pulse shown inset.

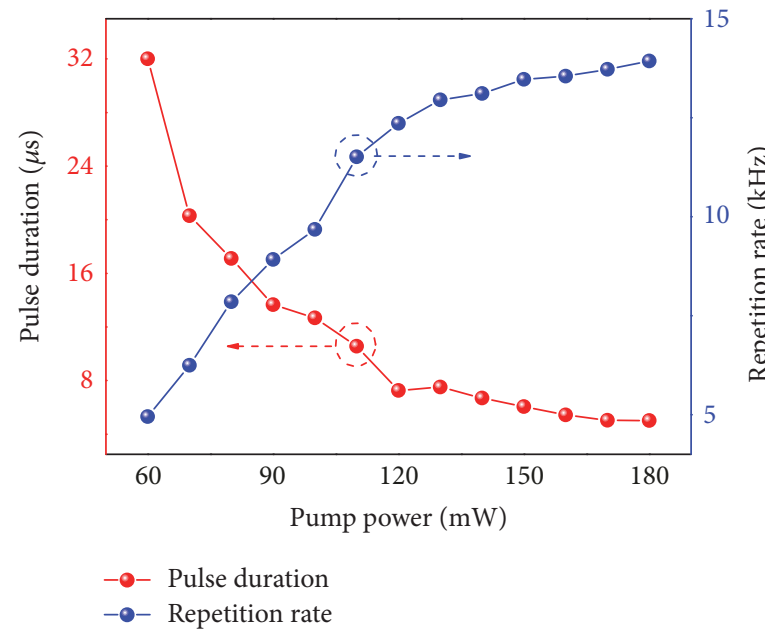

(a)

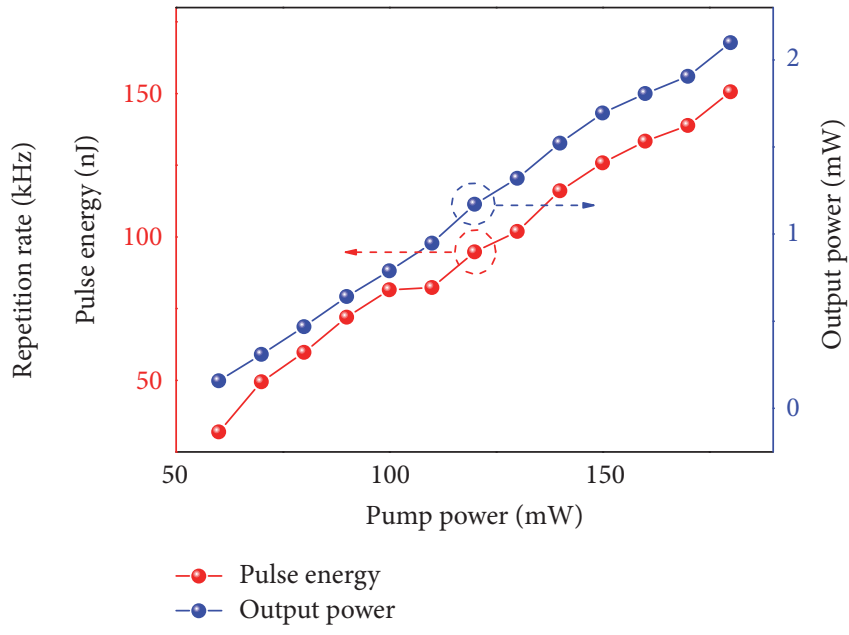

(b)

FIgURE 5: (a) The relationship between pulse duration and repetition rate with pump power. (b) The trend curves of output power and pulse energy with the pump power.

have strong evanescent field to interact with the material $[19,20]$, the waveguide will be easy to introduce large loss and strong nonlinearity for the small core size. However, the Dshaped fiber can provide a robust platform for the evanescent field interactions between light field propagating along the Dshaped fiber and the few-layer $\mathrm{MoTe}_{2}$.

\section{Conclusions}

We have successfully demonstrated an all-fiberized passively Q-switched EDFL by using an evanescent field interaction based $\mathrm{MoTe}_{2}$. The $\mathrm{MoTe}_{2}$ has been prepared via chemical vapor deposition method and then integrated with $\mathrm{D}$-shaped fiber to act as the nonlinear optical modulators. Maximum output pulse energy of $150.6 \mathrm{~nJ}$ has been obtained at a repetition rate of $13.9 \mathrm{kHz}$ from the laser cavity under the input pump power of $180 \mathrm{~mW}$ at $980 \mathrm{~nm}$. The experimental results can not only broaden the nonlinear application of
$\mathrm{MoTe}_{2}$, but provide an inroad for other TMDs nonlinear materials and their hybrid systems.

\section{Data Availability}

The experimental data used to support the findings of this study are available from the corresponding author upon request.

\section{Conflicts of Interest}

The authors declare that there are no conflicts of interests regarding the publication of this paper.

\section{Acknowledgments}

The authors would like to acknowledge Dr. Donglin Lu from Xiangtan University for providing the $\mathrm{MoTe}_{2}$ sample for the experiment. 


\section{References}

[1] F. Bonaccorso, Z. Sun, T. Hasan, and A. C. Ferrari, "Graphene photonics and optoelectronics," Nature Photonics, vol. 4, no. 9, pp. 611-622, 2010.

[2] M. E. Fermann and I. Hartl, "Ultrafast fibre lasers," Nature Photonics, vol. 7, no. 11, pp. 868-874, 2013.

[3] Q. Bao, H. Zhang, Y. Wang et al., "Atomic-layer craphene as a saturable absorber for ultrafast pulsed lasers," Advanced Functional Materials, vol. 19, no. 19, pp. 3077-3083, 2009.

[4] Z. Sun, T. Hasan, F. Torrisi et al., "Graphene mode-locked ultrafast laser," ACS Nano, vol. 4, no. 2, pp. 803-810, 2010.

[5] J. A. Alvarez-Chavez, H. L. Offerhaus, J. Nilsson, P. W. Turner, W. A. Clarkson, and D. J. Richardson, "High-energy, highpower ytterbium-doped Q-switched fiber laser," Optics Expresss, vol. 25, no. 1, pp. 37-39, 2000.

[6] S. Yamashita, Y. Inoue, S. Maruyama et al., "Saturable absorbers incorporating carbon nanotubes directly synthesized onto substrates and fibers and their application to mode-locked fiber lasers," Optics Expresss, vol. 29, no. 14, pp. 1581-1583, 2004.

[7] F. Wang, A. G. Rozhin, V. Scardaci et al., "Wideband-tuneable, nanotube mode-locked, fibre laser," Nature Nanotechnology, vol. 3, no. 12, pp. 738-742, 2008.

[8] Q. H. Wang, K. Kalantar-Zadeh, A. Kis, J. N. Coleman, and M. S. Strano, "Electronics and optoelectronics of two-dimensional transition metal dichalcogenides," Nature Nanotechnology, vol. 7, no. 11, pp. 699-712, 2012.

[9] S. Wang, H. Yu, H. Zhang et al., "Broadband few-layer $\mathrm{MoS}_{2}$ saturable absorbers," Advanced Materials, vol. 26, no. 21, pp. 3538-3544, 2014.

[10] H. Zhang, S. B. Lu, J. Zheng et al., "Molybdenum disulfide $\left(\mathrm{MoS}_{2}\right)$ as a broadband saturable absorber for ultra-fast photonics," Optics Express, vol. 22, no. 6, pp. 7249-7260, 2014.

[11] L. Du, G. Jiang, L. Miao et al., "Few-layer rhenium diselenide: an ambient-stable nonlinear optical modulator," Optical Materials Express, vol. 8, no. 4, p. 926, 2018.

[12] C. Zhao, H. Zhang, X. Qi et al., "Ultra-short pulse generation by a topological insulator based saturable absorber," Applied Physics Letters, vol. 101, p. 118, 2012.

[13] C. Zhao, Y. Zou, Y. Chen et al., "Wavelength-tunable picosecond soliton fiber laser with Topological Insulator: $\mathrm{Bi}_{2} \mathrm{Se}_{3}$ as a mode locker," Optics Express, vol. 20, no. 25, pp. 27888-27895, 2012.

[14] S. Chen, Q. Wang, C. Zhao, and Y. Li, "Stable singlelongitudinal-mode fiber ring laser using topological insulatorbased saturable absorber," Journal of Lightwave Technology, vol. 32, pp. 4438-4444, 2014.

[15] G. Jiang, Y. Jin, L. Miao et al., “Tunable Gold Nanorods QSwitcher for Pulsed Er-Doped Fiber Laser," IEEE Photonics Journal, vol. 9, no. 5, pp. 1-9, 2017.

[16] Z. Qin, G. Xie, H. Zhang et al., "Black phosphorus as saturable absorber for the Q-switched Er: ZBLAN fiber laser at $2.8 \mu \mathrm{m}$," Optics Express, vol. 23, no. 19, pp. 24713-24718, 2015.

[17] Y. Chen, G. Jiang, S. Chen et al., "Mechanically exfoliated black phosphorus as a new saturable absorber for both Q-switching and mode-locking laser operation," Optics Express, vol. 23, no. 10, pp. 12823-12833, 2015.

[18] D. Mao, B. Du, D. Yang et al., "Nonlinear Saturable Absorption of Liquid-Exfoliated Molybdenum/Tungsten Ditelluride Nanosheets," Small, vol. 12, no. 11, pp. 1489-1497, 2016.

[19] J. Wang, Z. Jiang, H. Chen et al., "High energy soliton pulse generation by a magnetron-sputtering-deposition-grown $\mathrm{Moe}_{2}$," Photonics Research, vol. 6, no. 6, pp. 535-541, 2018.
[20] M. Liu, W. Liu, P. Yan, S. Fang, H. Teng, and Z. Wei, "Highpower $\mathrm{MoTe}_{2}$-based passively Q-switched erbium-doped fiber laser," Chinese Optics Letters, vol. 16, no. 2, Article ID 020007, 2018.

[21] Z. Yan, T. Li, S. Zhao et al., "MoTe ${ }_{2}$ saturable absorber for passively Q-switched Ho,Pr:LiLuF 4 laser at $3 \mu \mathrm{m}$," Optics \& Laser Technology, vol. 100, pp. 261-264, 2018.

[22] M. Grzeszczyk, K. Gołasa, M. Zinkiewicz et al., "Raman scattering of few-layers $\mathrm{MoTe}_{2}$, " $2 \mathrm{D}$ Materials, vol. 3, no. 2, Article ID 025010, 2016. 

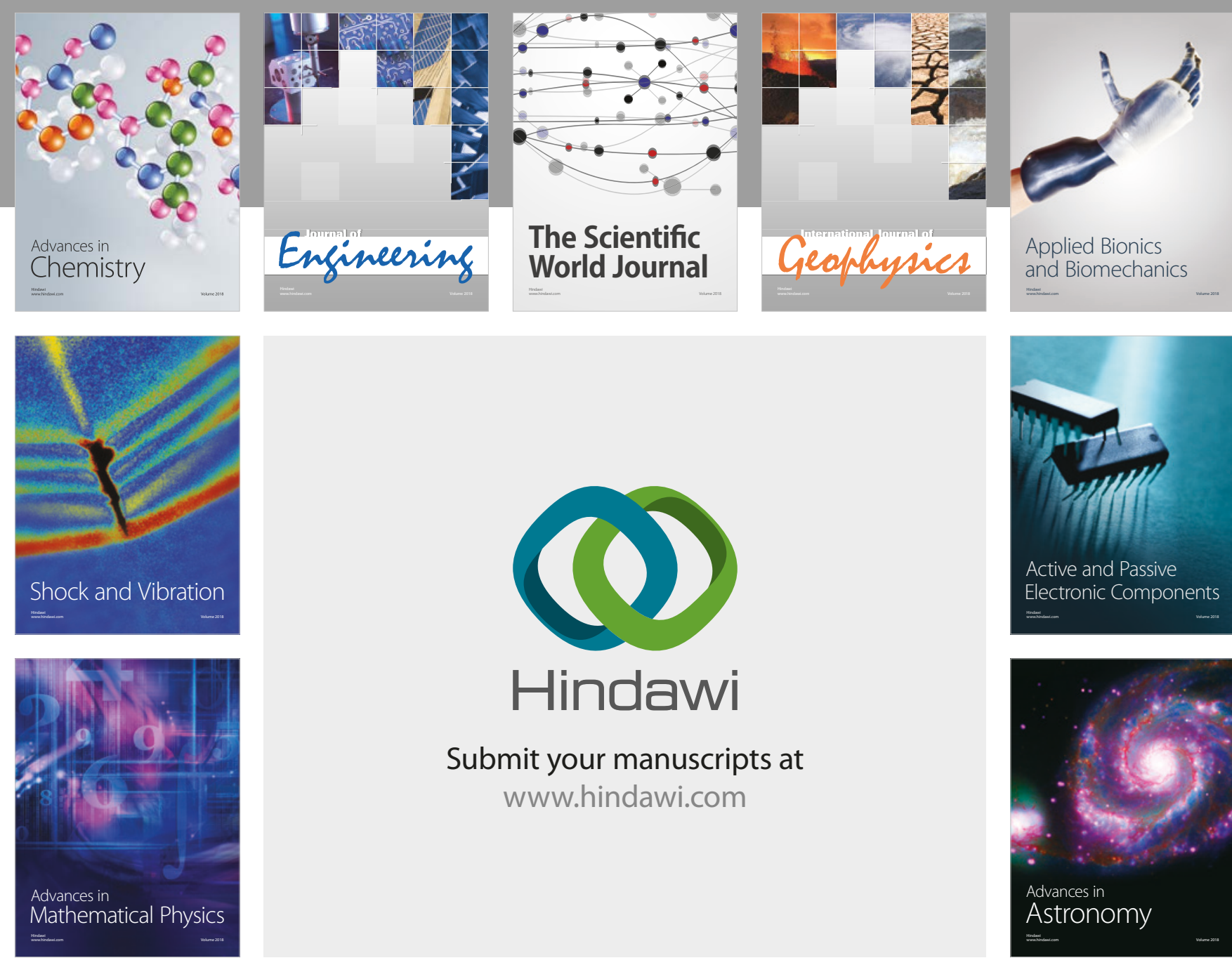

Submit your manuscripts at

www.hindawi.com

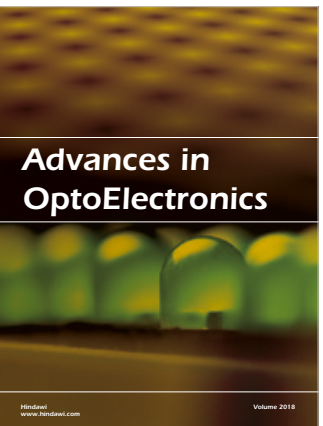

\section{Rotcting Machinery}
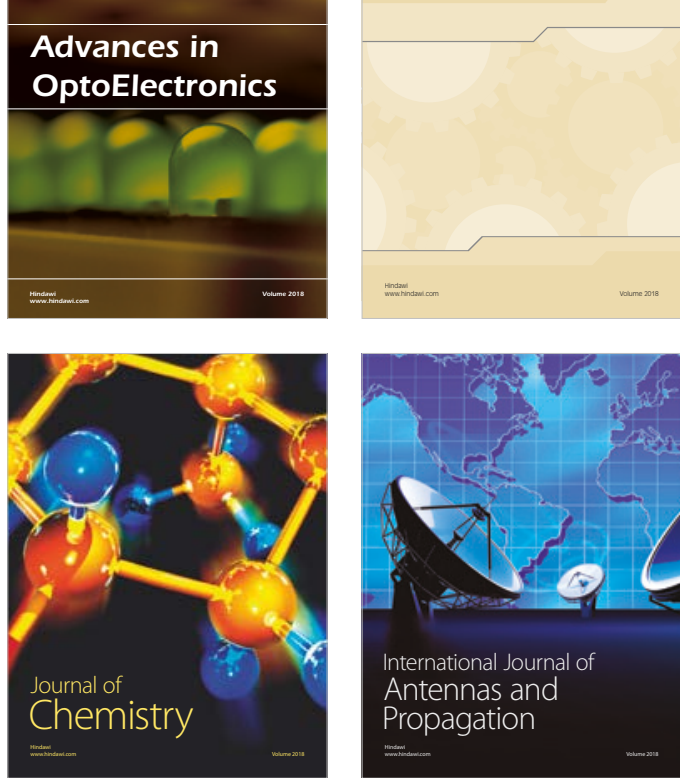

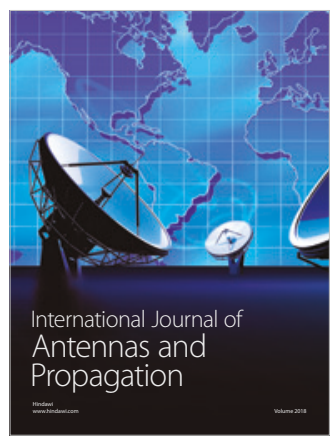

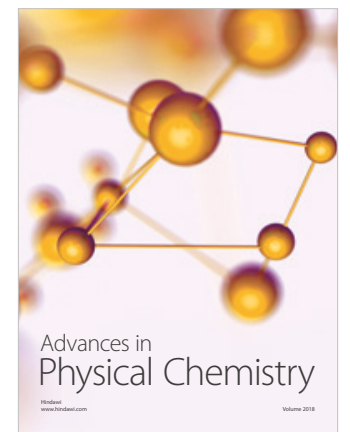

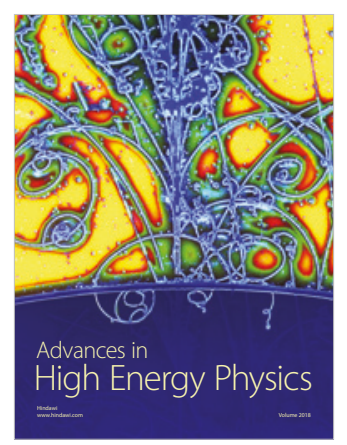

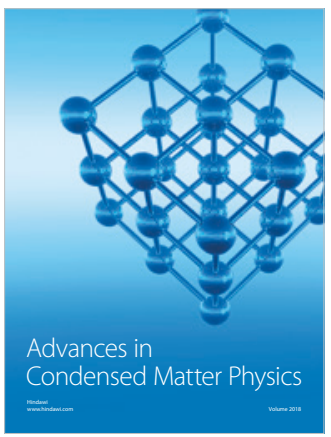

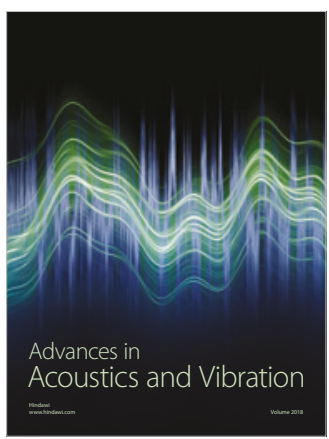

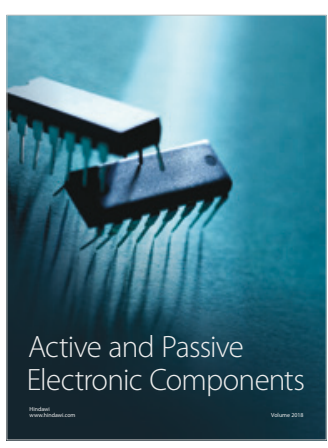
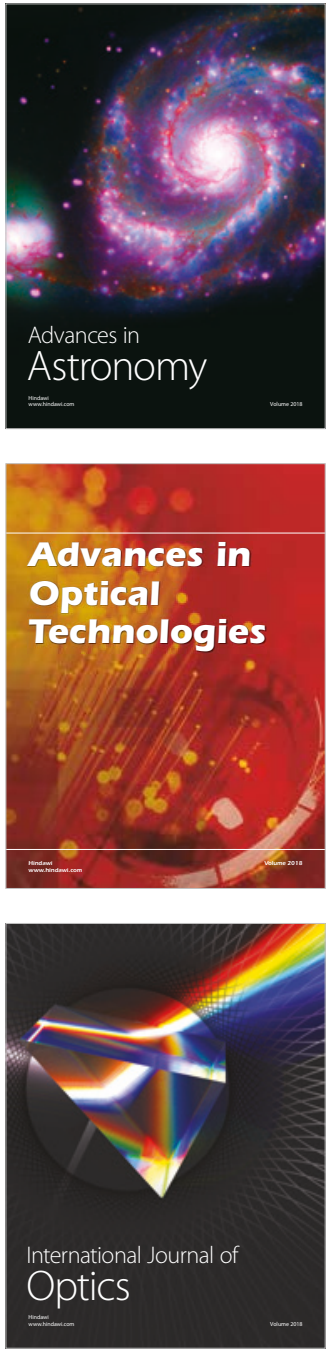